\title{
Title: Direct Comparison of Three PCR Methods in Detecting EGFR Mutations in Patients with Advanced Non-Small-Cell Lung Cancer
}

Takaya Ikeda, MD; Yoichi Nakamura, MD, PhD"; Hiroyuki Yamaguchi, MD, PhD; Nanae Tomonaga, $\mathrm{MD}^{1}$; Seiji Doi, MD, $\mathrm{PhD}^{2}$; Katsumi Nakatomi, MD, $\mathrm{PhD}^{1}$; Tetsuya lida, MD, PhD; Kohei Motoshima, MD; Kosuke Mizoguchi, MD; Takeshi Nagayasu, $\mathrm{MD}, \mathrm{PhD}^{3}$; Kazuhiro Tsukamoto, MD, $\mathrm{PhD}^{4}$; Shigeru Kohno, MD, $\mathrm{PhD}^{1}$

${ }^{1}$ Second Department of Internal Medicine, Nagasaki University School of Medicine, Nagasaki, Japan

${ }^{2}$ Department of Internal Medicine, Sasebo General Hospital, Nagasaki, Japan

${ }^{3}$ First Department of Surgery, Nagasaki University Hospital, Nagasaki, Japan

${ }^{4}$ Department of Pharmacotherapeutics, Nagasaki University Graduate School of Biochemical Science, Nagasaki, Japan

Running Title: Comparison of three PCR methods in the clinic

Key Words: non-small-cell lung cancer; EGFR mutations; mutant-enriched PCR;

PNA-LNA PCR clamp; PCR invader

Correspondence to: Yoichi Nakamura, MD, PhD

Second department of Internal Medicine, Nagasaki University Hospital, 1-7-1

Sakamoto, Nagasaki 852-8501, Japan

Tel: +81(95)-849-7273; Fax: +81(95)-849-7285; E-mail: yi-nakamu@umin.ac.jp 


\section{Micro abstract}

We compared three PCR methods (mutant-enriched PCR, PNA-LNA PCR and PCR clamp) to detect EGFR mutations in 50 patients with advanced NSCLC. Seventeen were harboring EGFR mutations, five of whom showed discrepancies between the results of different PCR methods. All five responded to gefitinib, which we consider to suggest that the discrepancies were false negatives.

\section{Clinical Practice Points}

- Several methods have been used to detect EGFR mutations in non-small-cell lung cancer (NSCLC): however, it is not clear which is the most suitable for use in the clinic.

- We compared three PCR methods (mutant-enriched PCR, PNA-LNA PCR and PCR clamp) in 50 patients with advanced NSCLC. Seventeen of the patients were harboring EGFR mutations, five of whom showed discrepancies between the results of different PCR methods. All five patients responded to gefitinib.

- We considered that all of the discrepancies might be false negatives because the patients responded to gefitinib. To clarify the reasons for the false negatives of each PCR method, and establish the clinical sensitivity and specificity of each method, a large prospective clinical trial is warranted. 


\section{Abstract}

Background: Epidermal growth factor receptor (EGFR) mutations are predictive of response to EGFR tyrosine kinase inhibitors (EGFR-TKIs) in non-small-cell lung cancer (NSCLC). Several methods have been used to detect EGFR mutations: however, it is not clear which is the most suitable for use in the clinic. In this study, we directly compare the clinical sensitivity and specificity of three PCR methods. Patients and Methods: We compared the three PCR methods (mutant-enriched PCR, PNA-LNA PCR and PCR clamp) in patients with advanced NSCLC. A patient who showed sensitive mutations by at least one PCR method was treated with gefitinib. A patient who showed no sensitive mutations was treated with chemotherapy with cytotoxic agents. Results: Fifty patients with advanced NSCLC previously untreated with EGFR-TKIs were enrolled in this trial. Seventeen of the patients were harboring EGFR mutations, five of whom showed discrepancies between the results of different PCR methods. All five patients responded to gefitinib. All patients harboring EGFR mutations received gefitinib treatment and 21 of 33 EGFR-mutation-negative patients received chemotherapy with cytotoxic agents. Median progression-free survival of the gefitinib group and the chemotherapy group were 8.2 months and 5.9 months, respectively. Conclusion: We considered that all of the discrepancies might be false negatives because the patients responded to gefitinib. To clarify the reason for the false negatives of each PCR method, and establish the clinical sensitivity and specificity of each PCR method, a large prospective clinical trial is warranted. 


\section{Introduction}

Gefitinib is an epidermal growth factor receptor tyrosine kinase inhibitor (EGFR-TKI) that competes with adenosine triphosphate (ATP) for the ATP-binding site of EGFR tyrosine kinase. ${ }^{1,2}$ Approximately $20-30 \%$ of patients with non-small-cell lung cancer (NSCLC) harbor EGFR gene mutations. ${ }^{3-5}$ Point mutations of exon18 and exon21, and deletions of exon19, are known to be sensitive for gefitinib. ${ }^{6}$ About $85-90 \%$ of these mutations are deletions of exon 19 and point mutations of exon21..$^{7-9}$ These EGFR mutations are predictive of response to EGFR-TKIs in patients with NSCLC. ${ }^{10}$ Recently, two randomized phase 3 trials revealed that first-line gefitinib treatment in patients with advanced NSCLC harboring EGFR mutations of exon19 or exon21 showed longer progression-free survival (PFS) with less toxicity compared with platinum doublet chemotherapy. ${ }^{11,12}$ Considering these results, EGFR gene status is most important in selecting anticancer drugs for patients with advanced NSCLC.

Clinical samples such as surgical specimens, lung biopsy and cytology ordinarily contain a few malignant cells and many normal cells. Thus, an assay is required to detect EGFR mutations against a large background of wild-type genes. Some polymerase chain reaction (PCR) methods have been used to detect EGFR mutations in the clinic. Mutant-enriched PCR is a highly sensitive PCR assay in two stages. The first stage entails amplification of mutant and wild-type sequences, followed by a selective restriction enzyme digestion of the wild-type sequence. The second stage involves amplification of the undigested mutant sequence. Kahn et al. reported detection of mutant K-ras genes using mutant-enriched $\mathrm{PCR}^{13}$ and Asano et al. developed the method to detect EGFR gene mutations in lung cancer. ${ }^{14}$ The peptide nucleic acid-locked nucleic acid (PNA-LNA) PCR clamp method has high sensitivity 
for detecting mutations through the use of PNA clamp primers that bind to the wild-type sequence and suppress their amplification. Nagai et al. have reported that the PNA-LNA PCR clamp method can detect EGFR mutations with high sensitivity. ${ }^{15}$ The PCR invader method detects mutations using cleavase enzyme that recognizes overlapping structures of the invader probes and mutation sequence. Previous authors have reported that the PCR invader method was useful for detecting polymorphisms and identifying genotypes. ${ }^{16,17}$ These three methods are known to be highly sensitive: their sensitivities are approximately the same. ${ }^{18}$

These PCR methods are in common clinical use; however, they have not been directly and prospectively compared with respect to clinical sensitivity and specificity. In this study, we directly compared these three PCR methods in the detection of EGFR mutations prospectively in patients with advanced NSCLC, and evaluated the false-positive and -negative rates in the clinic.

\section{Patients and methods}

\section{Eligibility criteria}

Patients who satisfied the following criteria were enrolled: stage IIIb or IV, and recurrence after operation; age $\geq 20$ years; Eastern Cooperative Oncology Group (ECOG) performance status (PS) $\leq 2$; adequate organ functions; and no medical problems to prevent compliance with the protocol. We obtained signed informed consent from patients with NSCLC who visited Nagasaki University Hospital, and analyzed their EGFR mutations using the mutant-enriched PCR, PNA-LNA PCR clamp and PCR invader methods. EGFR-sensitive-mutation-positive patients received gefitinib treatment and mutation-negative patients received chemotherapy with 
cytotoxic agents.

\section{Tumor Samples}

Samples were obtained from paraffin-embedded sections of specimens, frozen lung cytology specimens that were obtained from bronchoscopy (washing and brushing), or malignant pleural effusions. Each sample was divided into three parts, and each PCR method was performed. In the samples from paraffin-embedded sections, the micro-dissection method was not used.

\section{Mutant-enriched PCR method}

The deletion region in exon19 was amplified by PCR with forward primer: 5'-ATCCCAGAAGGTGAGAAAGATAAAATTC-3' and reverse primer: 5'-CCTGAGGTTCAGAGCCATGGA-3'. The PCR products were digested with Msel (New England BioLabs, Inc., Beverly, MA, USA). After the digest was amplified, the second PCR products were separated by $6 \%$ polyacrylamide gel electrophoresis (PAGE) and visualized by ethidium bromide staining. The point mutation region in exon21 was amplified by PCR using forward primer: 5'-CAGCCAGGAACGTACTGGTGA-3' and reverse primer: 5'-TCCTGGTGTCAGGAAAATGCT-3'. The PCR products were digested with Mscl (New England BioLabs, Inc.). After the digest was amplified, the second PCR products were digested with Asul (Fermentas International, Inc., Ontario, Canada). The digests were then subjected to separation by $8 \%$ PAGE and visualized by ethidium bromide staining. ${ }^{14,19-21}$ 


\section{PNA-LNA PCR clamp method}

The PNA-LNA PCR clamp method preferentially amplifies mutation sequences and detects mutations. The method needs peptide nucleic acid (PNA) clamp primer and locked nucleic acid (LNA) probe. PNA clamp primers bind to the wild-type sequence and suppress their amplification; LNA probes are designed to specifically detect mutant sequences and enhance their amplification in the presence of wild-type sequences, because PNA clamp primers competitively inhibit mutant LNA probes to bind to the wild type. ${ }^{15,22,23}$ To detect mutations of exon18 (G719C, G719S and G719A), exon19 \{E746-A750del (nt 2235-2249del), E746-A750 (nt 2236-2250), L747-A750del T751S, L747-S752del P753S, L747-E749del A750P, L747-S752del E746V and S752-1759del\} exon20 (T790M) and exon21 (L858R and L861Q), 12 probes were used. The PNA-LNA PCR clamp method was performed by Mitsubishi Kagaku Bio-chemical Laboratories Inc. (Tokyo, Japan).

\section{PCR invader method}

The invader method uses two oligonucleotide probes. An allele-specific single probe and an invader oligo probe hybridize to the single-stranded target DNA and form an overlapping structure. The $5^{\prime}$-flap of the single probe does not hybridize to the target DNA sequence. The 3'-end of the bound invader probe overlaps the primary probe by a single base of the mutation site. A cleavase enzyme recognizes this overlapping structure of the mutation site and cleaves the 5 '-flap of the primary probe at the base of the overlap. If the probe does not hybridize completely at the site of target sequence, no overlapping structure is formed and cleavase does not cleave the 5 '-flap of the primary probe so the target-specific product is not released. The 
target-specific 5'-flap oligonucleotides hybridize to fluorescent resonance energy transfer (FRET), leading to the formation of an overlapping structure that is recognized by the cleavase enzyme. When the FRET is cleaved, a fluorophore is

released from a quencher on the FRET and generates a fluorescence signal. ${ }^{16,17}$ Ten mutations of exon18 (G719A, G719C and G719S), exon19 \{E746-A750del (nt 2235-2249del), E746-A750del (nt 2236-2250del) and L747-P753del ins S\}, exon20 (S768I and T790M) and exon21 (L858R and L861Q) were determined. The PCR invader method was performed by BML (Tokyo, Japan).

\section{Treatment schedule}

EGFR-mutation-positive patients received gefitinib at a dose of $250 \mathrm{mg} /$ day. EGFR-mutation-negative patients received chemotherapy. Chemotherapy regimens were left to the discretion of the attending physician. Treatments were continued until disease progression or intolerable toxicities became apparent or the patient refused further treatment.

\section{Evaluation}

We defined EGFR-mutation-negative patients as those in whom no EGFR-mutations were detected by any of the three PCR methods, and EGFR-mutation-positive patients as those in whom an EGFR mutation was detected by one or more PCR method. Mutation-positive patents were treated with gefitinib even when there were discrepancies in the PCR results. We defined false negative as an EGFR-mutation-negative case that responded to gefitinib, and false positive as an EGFR-mutation-positive case that did not respond to gefitinib. ${ }^{24}$ 
The response was evaluated according to the Response Evaluation Criteria in Solid Tumors (RECIST). ${ }^{25}$ Adverse events were graded according to the Common Terminology Criteria for Adverse Events version 3.0 (CTCAE v3.0) (http://www.jcog.jp/doctor/tool/CTCAEv3J_guideline_041027_2.pdf). PFS was defined as the time from the date of beginning treatment to the date of disease progression or death. Overall survival (OS) was assessed from the date of beginning treatment until death from any cause.

\section{Statistical Analysis}

We analyzed differences in positive rate between tissue and cytology using Fisher's exact test. In EGFR-mutation-positive patients receiving gefitinib and EGFR-mutation-negative patients receiving chemotherapy, we analyzed PFS. PFS and OS were calculated by the Kaplan-Meier method, with differences between the groups compared using the log-rank test. A P-value $<0.05$ was considered statistically significant.

\section{Results}

From April 2008 to May 2010, 50 patients with advanced NSCLC previously untreated with EGFR-TKIs were enrolled in this study. Of these patients, 31 were men and 19 were women, with a median age of 68 years (range 24-89 years). Forty-seven of the patients had adenocarcinomas. Samples included 11 surgical specimens, 10 lung biopsies, 19 lung cytologies (washing and brushing), 5 pleural effusions and 5 lymph-node biopsies (Table 1). All samples were confirmed to contain malignant cells. 
mutant-enriched method, 16 samples showed positive; the PNA-LNA PCR clamp method showed 14 positive samples; and the PCR invader method showed 15 positive samples (Table 2). There were five cases that showed discrepancies in their result. The mutant-enriched method showed one of these five cases to be negative: the other two methods showed two negatives, respectively. In case 4 , the mutant-enriched method and the PNA-LNA PCR clamp method showed mutations in exon19 and 21, but the PCR invader method showed only the exon19 mutation (Table 3). In the 47 samples of adenocarcinoma, the positive rate was $46.2 \%$ (12 of 26) in tissue samples and $23.8 \%$ (5 of 21$)$ in cytological samples, respectively. There was no statistically significant difference between them (Table 4).

Seventeen patients harboring EGFR mutations were assigned to receive gefitinib and 33 patients without EGFR mutations were assigned to receive chemotherapy with cytotoxic agents. However, five patients were excluded from the chemotherapy group because of poor PS and seven were excluded because they received other treatments. Of the 21 patients who received chemotherapy, 15 received a platinum-containing regimen and 6 a single-agent regimen (Fig. 1).

Of the 17 patients who received gefitinib, 15 patients $(88.2 \%)$ exhibited partial response and 2 exhibited stable disease. All five patients who showed discrepancies between the three PCR methods exhibited partial response. PFS was significantly longer in the gefitinib group than in the chemotherapy group (median 8.2 months vs. 5.9 months; HR 0.457; 95\% Cl 0.211-0.990; P=0.0472) (Fig. 2).

In the gefitinib group, the most common adverse events were grade 1 or 2 skin rash and grade 1 paronychia. One instance of grade 3 alanine aminotransferase/aspartate aminotransferase elevation was observed. Interstitial lung disease was reported in one patient. In the chemotherapy group, toxicities $>$ grade3 
were neutropenia (33.3\%), leucopenia (14.3\%), thrombocytopenia $(4.7 \%)$, oral mucositis $(4.7 \%)$, corneal ulcer $(4.7 \%)$, and hyponatremia $(4.7 \%)$. While these toxicities seem severe compared with those associated with gefitinib treatment, they are comparable to previous reports of cytotoxic agents. There were no treatment-related deaths in the gefitinib group.

\section{Discussion}

In this study, we directly compared the clinical sensitivity of three PCR methods in patients with advanced NSCLC. Seventeen patients were harboring sensitive EGFR gene mutations, and five of them showed discrepancies from the three methods. In this study, all samples were histopathologically confirmed to contain malignant cells and as all five patients responded to gefitinib treatment, we considered that these discrepancies were all caused by false negatives.

Recently, two randomized phase 3 trials revealed that the efficacy of first-line gefitinib was superior to that of standard chemotherapy in patients with advanced NSCLC harboring sensitive EGFR mutations. ${ }^{11,12}$ Consequently, gefitinib has become adopted as first-line treatment for patients with advanced NSCLC harboring sensitive EGFR mutations in Japan. ${ }^{26}$ Compared with platinum-based chemotherapy, the toxicities of gefitinib are mild and acceptable. ${ }^{11,12}$ If false-positive patients are treated with gefitinib, the patients seldom suffer severe toxicities although the tumor does not respond. On the contrary, if false-negative patients are treated with platinum-based chemotherapy, there are severe toxicities and less clinical benefits. In addition, once the patients are diagnosed as not harboring sensitive EGFR mutations, physicians might not treat them with EGFR-TKIs even after the relapse of first-line chemotherapy, though second-line gefitinib was previously reported to show acceptable toxicity and 
efficacy for patients with NSCLC harboring EGFR-sensitive mutations. ${ }^{27,28}$ Thus, a false negative seems to be a more severe problem for patients with NSCLC harboring EGFR-sensitive mutations.

At the beginning of this trial, we expected that detection of EGFR gene mutations in the clinic might give false positives but not as false negatives, because these PCR methods were reported to have high sensitivity. The mutant-enriched PCR method can detect EGFR mutations in the presence of 500-fold background levels of wild-type EGFR in normal cells. ${ }^{14}$ The PNA-LNA PCR clamp method is reported to be able to detect a mutant EGFR sequence in specimens containing 100 to 1000 excess copies of wild-type EGFR sequence. ${ }^{15,29}$ Naoki et al. have reported that the PCR-invader method can detect a known EGFR mutation in lung cancer cells at 100-1000-fold dilution. ${ }^{30}$ Thus, we supposed that false negatives seldom arise in these PCR methods: however, we detected five false negatives in the 17 patients harboring EGFR mutations. The causes of the false negatives were unclear in this trial. Additionally, it was difficult to conduct the statistical analysis between PCR methods because discrepancies were detected in only five patients and the trial had a small sample size. However, the rate of false negative was high compared with previous reports. ${ }^{30}$ A prospective clinical mega-trial is needed to investigate what causes the false negatives in each PCR method.

\section{Conclusions}

We prospectively compared the clinical sensitivity of three PCR methods to analyze EGFR mutations, and detected discrepancies in the results in five patients. We suggest that all of these discrepancies might be false negatives. To clarify the reason for the false negatives in each PCR methods, and establish the clinical 
sensitivity and specificity of each PCR method, a prospective clinical trial is needed.

\section{Conflict of Interest Statement}

None declared. 


\section{References}

1. Ono M, Hirata A, Kometani T, et al. Sensitivity to gefitinib (Iressa, ZD1839) in non-small cell lung cancer cell lines correlates with dependence on the epidermal growth factor (EGF) receptor/extracellular signal-regulated kinase $1 / 2$ and EGF receptor/Akt pathway for proliferation. Mol Cancer Ther 2004; 3:465-72.

2. Han SW, Hwang PG, Chung DH, et al. Epidermal growth factor receptor (EGFR) downstream molecules as response predictive markers for gefitinib (Iressa, ZD1839) in chemotherapy-resistant non-small cell lung cancer. Int $J$ Cancer 2005; 113:109-15.

3. Shigematsu H, Lin L, Takahashi T, et al. Clinical and biological features associated with epidermal growth factor receptor gene mutations in lung cancers. J Natl Cancer Inst 2005; 97:339-46.

4. Paez JG, Jänne PA, Lee JC, et al. EGFR mutations in lung cancer: correlation with clinical response to gefitinib therapy. Science 2004; 304:1497-500.

5. Mitsudomi T, Kosaka T, Endoh H, et al. Mutations of the epidermal growth factor receptor gene predict prolonged survival after gefitinib treatment in patients with non-small-cell lung cancer with postoperative recurrence. J Clin Oncol 2005; 23:2513-20.

6. Han SW, Kim TY, Hwang PG, et al. Predictive and prognostic impact of epidermal growth factor receptor mutation in non-small-cell lung cancer patients treated with gefitinib. J Clin Oncol 2005; 23:2493-501.

7. Kosaka $\mathrm{T}$, Yatabe $\mathrm{Y}$, Endoh $\mathrm{H}$, et al. Mutations of the epidermal growth factor receptor gene in lung cancer: biological and clinical implications. Cancer Res 2004; 64:8919-23.

8. Tokumo M, Toyooka S, Kiura K, et al. The relationship between epidermal growth factor receptor mutations and clinicopathologic features in non-small cell lung cancers. Clin Cancer Res 2005; 11:1167-73.

9. Mitsudomi T, Yatabe Y. Mutations of the epidermal growth factor receptor gene and related genes as determinants of epidermal growth factor receptor tyrosine kinase inhibitors sensitivity in lung cancer. Cancer Sci 2007; 98:1817-24.

10. Mitsudomi T, Kosaka T, Yatabe Y. Biological and clinical implications of EGFR mutations in lung cancer. Int J Clin Oncol 2006; 11:190-8.

11. Mitsudomi T, Morita S, Yatabe $Y$, et al. Gefitinib versus cisplatin plus docetaxel in patients with non-small-cell lung cancer harbouring mutations of the epidermal growth factor receptor (WJTOG3405): an open label, randomised phase 3 trial. Lancet Oncol 2010; 11:121-8.

12. Maemondo M, Inoue A, Kobayashi K, et al. Gefitinib or chemotherapy for non-small-cell lung cancer with mutated EGFR. N Engl J Med 2010; 362:2380-8.

13. Kahn SM, Jiang W, Culbertson TA, et al. Rapid and sensitive nonradioactive detection of mutant K-ras genes via 'enriched' PCR amplification. Oncogene 1991; 6:1079-83. 
14. Asano H, Toyooka S, Tokumo M, et al. Detection of EGFR gene mutation in lung cancer by mutant-enriched polymerase chain reaction assay. Clin Cancer Res 2006; 12:43-8.

15. Nagai $\mathrm{Y}$, Miyazawa $\mathrm{H}$, Huqun, et al. Genetic heterogeneity of the epidermal growth factor receptor in non-small cell lung cancer cell lines revealed by a rapid and sensitive detection system, the peptide nucleic acid-locked nucleic acid PCR clamp. Cancer Res 2005; 65:7276-82.

16. Lyamichev V, Mast AL, Hall JG, et al. Polymorphism identification and quantitative detection of genomic DNA by invasive cleavage of oligonucleotide probes. Nat Biotechnol 1999; 17:292-6.

17. Tadokoro K, Kobayashi M, Yamaguchi T, et al. Classification of hepatitis B virus genotypes by the PCR-Invader method with genotype-specific probes. $J$ Virol Methods 2006; 138:30-9.

18. Kimura $\mathrm{H}$, Kasahara $\mathrm{K}$, Kawaishi $\mathrm{M}$, et al. Detection of epidermal growth factor receptor mutations in serum as a predictor of the response to gefitinib in patients with non-small-cell lung cancer. Clin Cancer Res 2006; 12:3915-21.

19. Inukai M, Toyooka S, Ito S, et al. Presence of epidermal growth factor receptor gene T790M mutation as a minor clone in non-small cell lung cancer. Cancer Res 2006; 66:7854-8.

20. Soh J, Toyooka S, Aoe K, et al. Usefulness of EGFR mutation screening in pleural fluid to predict the clinical outcome of gefitinib treated patients with lung cancer. Int J Cancer 2006; 119:2353-8.

21. Yamaguchi $H$, Soda $H$, Nakamura $Y$, et al. Serum levels of surfactant protein D predict the anti-tumor activity of gefitinib in patients with advanced non-small cell lung cancer. Cancer Chemother Pharmacol 2011; 67:331-8.

22. Tanaka $\mathrm{T}$, Nagai $\mathrm{Y}$, Miyazawa $\mathrm{H}$, et al. Reliability of the peptide nucleic acid-locked nucleic acid polymerase chain reaction clamp-based test for epidermal growth factor receptor mutations integrated into the clinical practice for non-small cell lung cancers. Cancer Sci 2007; 98:246-52.

23. Sutani A, Nagai Y, Udagawa K, et al. Gefitinib for non-small-cell lung cancer patients with epidermal growth factor receptor gene mutations screened by peptide nucleic acid-locked nucleic acid PCR clamp. Br J Cancer 2006; 95:1483-9.

24. Mok TS, Wu YL, Thongprasert S, et al. Gefitinib or carboplatin-paclitaxel in pulmonary adenocarcinoma. N Engl J Med 2009; 361:947-57.

25. Therasse P, Arbuck SG, Eisenhauer EA, et al. New guidelines to evaluate the response to treatment in solid tumors. European Organization for Research and Treatment of Cancer, National Cancer Institute of the United States, National Cancer Institute of Canada. J Natl Cancer Inst 2000; 92:205-16.

26. Hayakawa K, Tsuboi M. Clinical Practice Guideline for lung cancer. Gan To Kagaku Ryoho 2010; 37:609-12.

27. Maruyama R, Nishiwaki Y, Tamura T, et al. Phase III study, V-15-32, of gefitinib versus docetaxel in previously treated Japanese patients with non-small-cell lung cancer. J Clin Oncol 2008; 26:4244-52. 
28. Kim ES, Hirsh V, Mok T, et al. Gefitinib versus docetaxel in previously treated non-small-cell lung cancer (INTEREST): a randomised phase III trial. Lancet 2008; 372:1809-18.

29. Tanaka T, Matsuoka M, Sutani A, et al. Frequency of and variables associated with the EGFR mutation and its subtypes. Int J Cancer 2010; 126:651-5.

30. Naoki K, Soejima K, Okamoto H, et al. The PCR-invader method (structure-specific 5' nuclease-based method), a sensitive method for detecting EGFR gene mutations in lung cancer specimens; comparison with direct sequencing. Int J Clin Oncol 2011; 16:335-44. 


\section{Figure legends}

Figure 1. Study Profile.

Figure 2. Progression-free Survival of Patients in the Present Study. Kaplan-Meier curves for progression-free survival. Tick marks indicate patients for whom data were censored at the data cutoff point. 


\section{Table 1}

Patient Characteristics $(n=50)$

\begin{tabular}{|c|c|}
\hline & No. of Patients \\
\hline Age, years & \\
\hline Median (Range) & $68(24-89)$ \\
\hline Sex & \\
\hline Male & 31 \\
\hline Female & 19 \\
\hline Histology & \\
\hline $\mathrm{Ad}$ & 47 \\
\hline AdSq & 1 \\
\hline Lr & 1 \\
\hline PD & 1 \\
\hline Samples & \\
\hline SS & 11 \\
\hline LB & 10 \\
\hline Cy & 19 \\
\hline PE & 5 \\
\hline LN & 5 \\
\hline
\end{tabular}

Ad, adenocarcinoma; Sq, squamous-cell carcinoma; AdSq, adenosquamous-cell carcinoma; Lr, large-cell carcinoma; PD, poorly differentiated carcinoma; SS, surgical specimen; LB, lung biopsy; Cy, lung cytology (washing and brushing); PE, pleural effusion; LN, lymph-node biopsy. 
Table 2.

Results of the Three Methods for Detecting EGFR Mutations

\begin{tabular}{l|c|c|c|c}
\hline \multicolumn{5}{c}{ Mutations } \\
\hline Method & Exon 19 & Exon 21 & Exon 19+21 & Total \\
\hline Enriched & 6 & 9 & 1 & 16 \\
Clamp & 4 & 9 & 1 & 14 \\
Invader & 5 & 10 & 0 & 15 \\
\hline Any of three & 6 & 10 & 1 & 17 \\
methods & & & & \\
\hline
\end{tabular}

Enriched, mutant-enriched PCR; Clamp, PNA-LNA PCR clamp; Invader, PCR invader. 
Table 3.

Characteristics of Five Patients Showing Discrepancy of Mutations

\begin{tabular}{c|c|c|c|c|c}
\hline $\begin{array}{c}\text { Cas } \\
\text { e }\end{array}$ & $\begin{array}{c}\text { Sampl } \\
\mathrm{e}\end{array}$ & Enriched & Clamp & Invader & Type of mutation \\
\hline 1 & SS & 19 & - & 19 & E746-A750del \\
2 & Cy & 19 & - & - & E746-A750del \\
3 & LB & 19 & 19 & - & E746-A750del \\
4 & SS & 19,21 & 19,21 & 19 & E746-A750del, L858R \\
5 & SS & - & Unextracted & 21 & L858R \\
\hline
\end{tabular}

SS, surgical specimen; Cy, lung cytology; LB, lung biopsy; Enriched, mutant-enriched PCR; Clamp, PNA-LNA PCR clamp; Invader, PCR invader. 
Table 4.

Comparison of Sample Type in Adenocarcinoma $(n=47)$

\begin{tabular}{c|c|c|c}
\hline Sample & Total & Mutation positive & $p$-value \\
\hline Tissue & 26 & $12(46.2 \%)$ & 0.376 \\
Cytology & 21 & $5(23.8)$ & \\
\hline
\end{tabular}


Figure 1.

\begin{tabular}{|c|}
\hline $\begin{array}{c}\text { NSCLC patients } \\
\mathrm{n}=50\end{array}$ \\
\hline
\end{tabular}

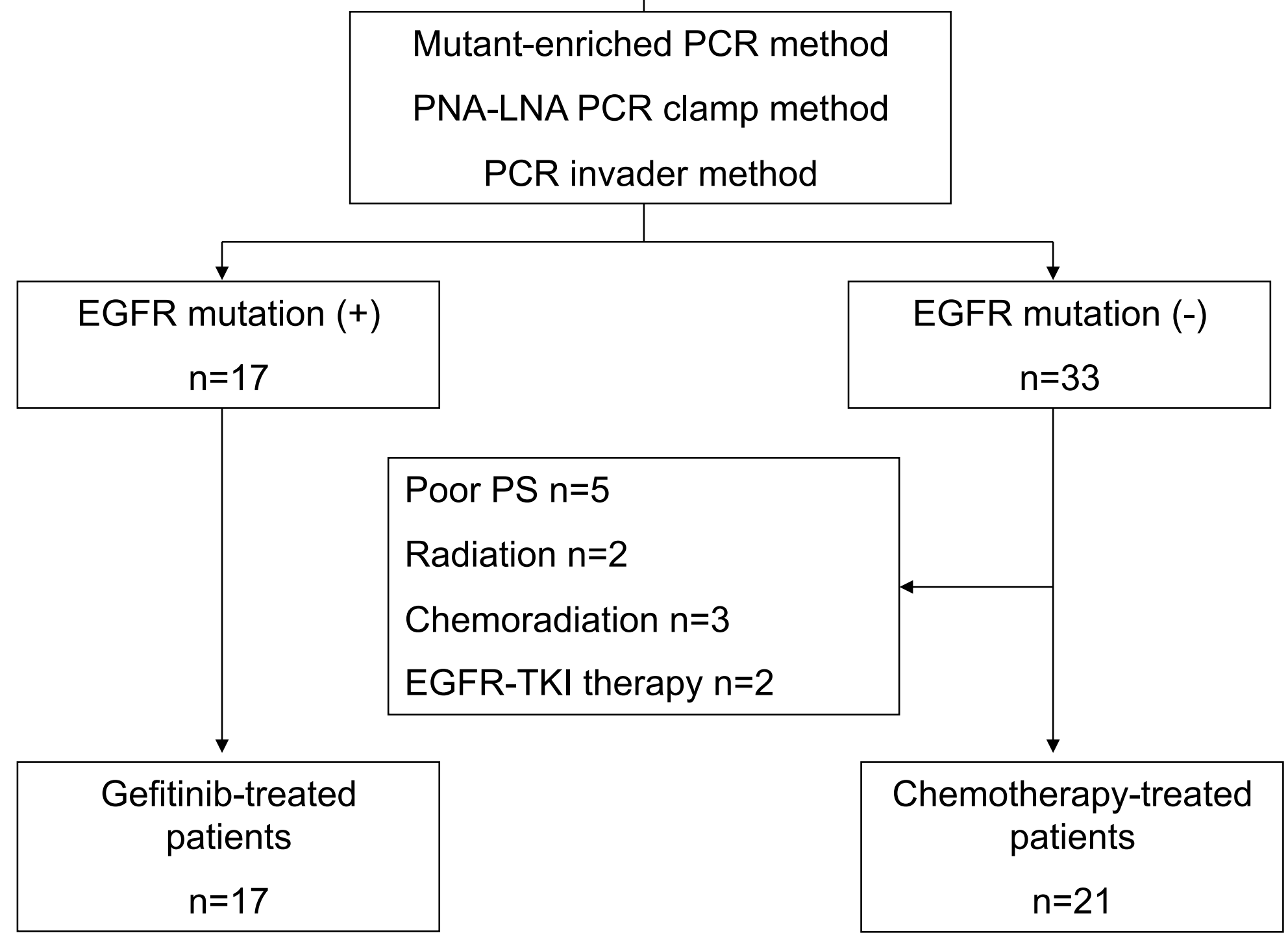


Figure 2.

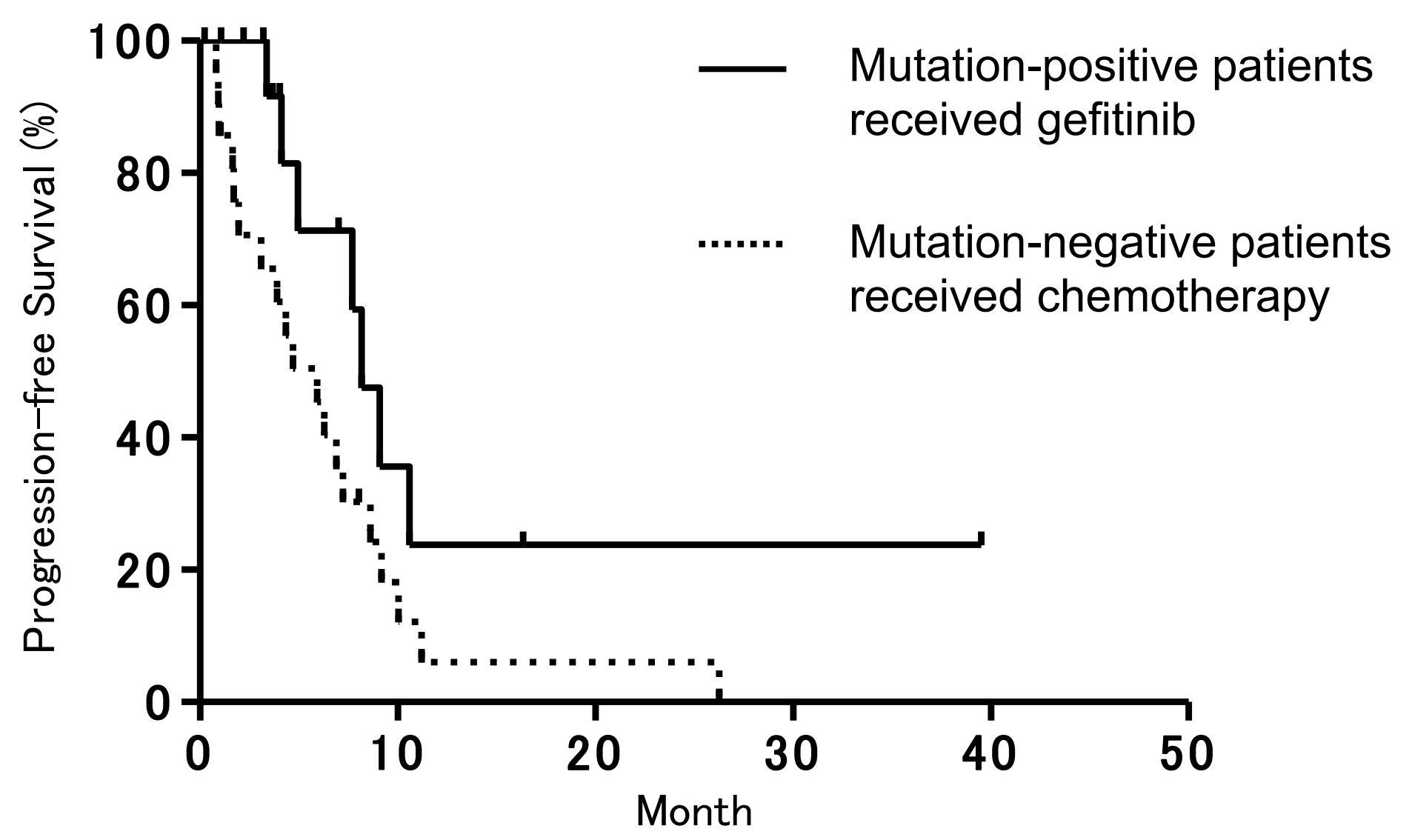

